\title{
Application of Log-polar Coordinate Transform in Image Processing
}

\author{
Yuping Feng ${ }^{1,}$, Shuang Chen ${ }^{1, b}$, Xuefeng Liu $^{1, c}$ \\ ${ }^{1}$ College of Automation and Electronic Engineering, Qingdao University of Science and Technology, \\ Qingdao, China \\ ajgjsfengyuping@163.com, bchenshuang_wf@hotmail.com, cnina.xf.liu@hotmail.com
}

Keywords: Log-polar coordinate; Image registration; Fourier-Mellin transform; Image compression

\begin{abstract}
Two kinds of application of log-polar coordinate transform based on human visual structure model in image processing are introduced: image registration and image compression. New solutions and improved measures are proposed in these two areas. In the field of image registration, improvement measures are proposed for Fourier-Mellin transform based on log-polar coordinate, experimental results show that the correlation peak by improved method is higher. In the field of image compression, the description method of image based on log-polar coordinate is proposed, which is a variable resolution storage and compression method, experimental results show that on the premise of not losing the details of the interest region interest, this variable resolution compression method can achieve the compression of $81 \%$ and achieve a large proportion of compressed storage of image with a certain purpose.
\end{abstract}

\section{Introduction}

The log-polar coordinate transform (LPT) model [1,2] is quite similar to human retinal structure. The distribution of the pyramidal cells in the retina is not uniform. The central is dense, while the peripheral is sparse. The resolution is smaller and smaller exponentially. So the visual system samples the external light signal with a non-liner logarithmic compression method. When one focuses something, the object being observed is very clear, while the surroundings are relatively blurred. Therefore the human eyes can quickly adapt to the target rotate or scale. The LPT has a lot of important applications in image processing, such as moving object attitude detection [3], image registration [4,5], image compression and storage etc..

In the field of image registration, the Fourier-Mellin transform (FMT) algorithm combined with log-polar coordinate model can make registration with the image of translation, rotation and scaling transformation. This method based on frequency domain utilizes all content in an image, and have a certain immunity to illumination, noise, local motion objects and fuzzy image. It has hardware support and fast algorithm (FFT), and it is a method commonly used in image registration.

In the field of image compression, such as in the military target tracking, wide field of view and high resolution can bring more high combat effectiveness, but it also carries a heavy burden to the computer, particularly missile or airborne computer, storage space is limited, the huge amount of data has increased storage burden, so the image data are necessarily compressed. Common and traditional compression methods [6,7] are designed for the whole image compression, compression ratio is not very high, considering in this particular environment, then we really only interested in the target tracking, and the background information is not particularly concerned, so the traditional compression methods is not appropriate.

Based on the above consideration, this paper introduces two kinds of application log-polar transform human structure model in image processing: image registration and image compression, and new solutions are proposed in these two areas. In the field of image registration, improvement measures of FMT based on log-polar coordinate are put forward, which makes the phase correlation peak is higher. In the field of image compression, based on log-polar coordinate sampling, a variable resolution storage and compression method of the region of interest image based on log-polar coordinate is presented, which can ensure not losing the details and reducing the resolution of the 
image. This compression method can compress image by a high compression ratio which is suitable for target tracking area.

\section{The principle of log-polar transform}

The LPT model is quite similar to human retinal structure. The resolution of the pyramidal cells in the central recess is the highest, the resolution exponentially decreases with the distance increasing between to the central recess, which is shown in Fig. 1 (a). When the eye gazes, the observation target is very clear, and the external background is fuzzy, so the human eyes can quickly adapt to the target rotate or scale. The LPT converts images from Cartesian coordinate system to polar coordinate system exactly in the way where the resolution is from high to low and the position is from center to surrounding, which is shown in Fig. 1.

The formula is as follows:

$$
\left\{\begin{array}{l}
u=k \ln \rho=k \ln \left(\sqrt{\left(x-x_{0}\right)^{2}+\left(y-y_{0}\right)^{2}}\right) \\
v=k \theta=k \arctan \left(\left(y-y_{0}\right) /\left(x-x_{0}\right)\right)
\end{array}\right.
$$

Where $(x, y)$ is the coordinate of the image in the Cartesian coordinate, $(u, v)$ is the coordinate after the LPT, $\left(x_{0}, y_{0}\right)$ is the center of coordinate transform, which is usually the image center. Considering the image will become very short and wide after LPT, the coordinate axis needs appropriate magnification, the magnification parameter, $k$ is introduced. The range of $\theta$ is $0 \square 2 \pi$, The formula for the inverse transformation is given by:

$$
\begin{aligned}
& x=x_{0}+e^{u / k} \cos (v / k) \\
& y=y_{0}+e^{u / k} \sin (v / k)
\end{aligned}
$$

According to Eq. (2), bilinear interpolation should be used for the implementation of the log-polar transformation. For example, if the log-polar transform is applied to an image with $256 \times 256$ pixels, the magnification factor is set to $k=30$, and the center of coordinate transform is set to $\left(x_{0}, y_{0}\right)=(129,129)$, then the transformed image in log-polar coordinate system is shown in Fig. $1(\mathrm{~d})$ with $146 \times 188$ pixels, where $u$ is the horizontal axis and $v$ is the vertical axis.

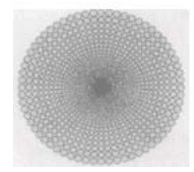

(a)

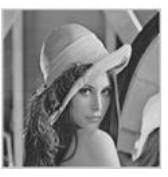

(b)

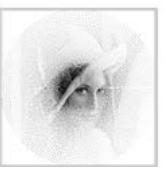

(c)

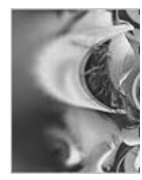

(d)

Fig. 1 (a)Distribution of human pyramidal cells (b) Cartesian coordinate image (c)Log-polar sampling image (d) Image in log-polar coordinate

When the original image in Cartesian coordinate, relative to the center of coordinate transform, is rotated $\mathrm{M}$ radian and amplified $\mathrm{N}$ times, there is:

$$
\begin{aligned}
& u^{\prime}=k \ln (N \rho)=k \ln N+k \ln \rho=u+k \ln N \\
& v^{\prime}=k(\theta+M)=k \theta+k M=v+k M
\end{aligned}
$$

It can be concluded that after log-polar coordinate transform, the scaling and rotation in Cartesian coordinate system become the translation in u direction and v direction, respectively, as shown in Fig. 2. The image in Cartesian coordinate is translation invariant, while the image in log-polar coordinate is rotation invariant. Using this property, we can calculate rotation and scaling parameters in the Cartesian coordinate system.

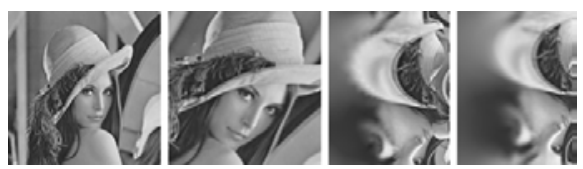

Fig. 2 Image rotation and scaling become translation in log-polar coordinate 


\section{Application of log-polar coordinate in image registration}

\section{Fourier-Mellin transform based on the LPT}

For the log-polar transform through image, it can carry out registration of two images with rotation and scaling, but the most important question to the registration method is the choice of the coordinate origin, namely the same point must be choose as the origin of coordinates transform when building log-polar coordinate, otherwise the LPT is not rotation invariant. If there is translation, rotation and scaling between two images at the same time, Fourier-Mellin transform should be used to solving the coordinate origin selection problem by utilizing the amplitude-frequency characteristics of images and establishing a log-polar coordinate system with the zero point on the amplitude spectrum used as the origin [8].

The Steps of image registration by FMT are as follows: apply log-polar transformation to the spectrum amplitude of images 1 and 2, and then calculate the rotation angle and scaling factor using the phase correlation method [9], then take inverse transformation to the image 1 using the calculated rotation angle and scaling factor, resulting a image 3, apply the phase correlation method again for 1 and 3 to calculating translation amount. According to the translation, rotation, scaling parameters, coordinate transformation matrix of 1 and 2 can be constructed, and registration and stitch can be realized by using this matrix.

\section{Improvement measures}

We need calculating spectrum amplitude and the log-polar transform in the FMT algorithm, some improvement for this process are suggested, they are as follows:

(1) If we directly take Fourier transform to image, a "+" shape characteristic will generate in frequency spectrum, which may bring false peaks in phase correlation method and affect the matching. Here we adopt window function [10] weighting to the image for removing the "+" shape characteristic and false peak.

(2) The frequency spectrum in 45 degree, 135 degree, 225 degree and 315 degree angle domain is more than the other angle domain after applying log-polar coordinate transform to the frequency spectrum, which will interfere the following the correlation detection. So we select the half of image length as the maximum log radius. The frequency spectrum will be the same in 360 degree range, and has a higher phase correlation peak.

\section{The registration experiments}

By using the improved method presented in the paper, we register two images which contain rotation and scaling transform, as shown in Fig. 3. It can be seen that phase correlation function of frequency spectrum in log-polar coordinate is more smooth, and the peak is obviously higher than before improvement.
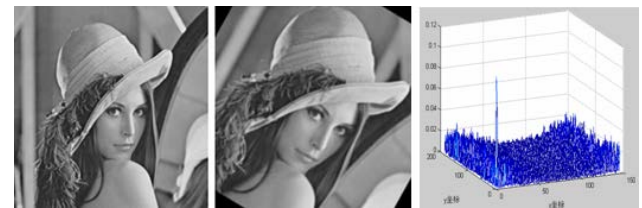

Fig. 3 (a) Images and phase correlation peak of frequency spectrum in log-polar coordinate

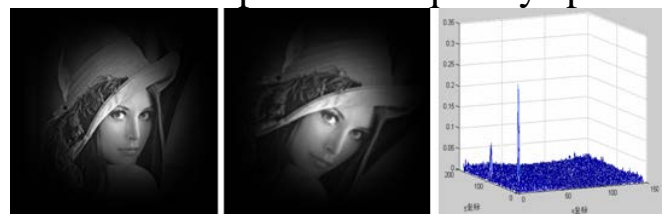

Fig. 3 (b) Improved image and phase correlation peak of frequency spectrum log-polar coordinate

\section{Application of log-polar in variable resolution image compression}

\section{The image compression principle in log-polar coordinate}

In the field of military target tracking, wide field of view and high resolution can bring more high combat effectiveness, but it also causes the computer to a heavy burden, the huge amount of data has increased the use of storage burden, a large proportion of the image data compression is urgent. Considering in the specific environment, we only need to be interest in the tracking of the target, and 
the background information is not particularly concerned, so traditional compression method is not particularly applicable.

Enlightened by the human visual structure, the distribution of the pyramidal cells in the retina is not uniform, the central is dense and peripheral is sparse. This characteristic makes that the visual information acquisition is non-uniform, the effective visual field is very big, but the clear identification of the field is only in the point of gaze around $6 \sim 8$ degree range. Thereby visual system can obtain high resolution information on the region of interest, and maintain a large field of view, and put on alert to the surrounding environment. The eyes can make the region of interest in the central recess constantly through the continuous rotation of the eyes.

Referring the processing mode of the human eyes, a various resolution image storage system can be used for compressing video image. The log-polar sampling transform is adopted through taking the region of interest as the origin, and it will keep high resolution for the interested tracking point. With the distance between to the region of interest, the resolution of surrounding area exponentially decreases. This method is especially suitable for storage of tracking effect.

The transformation relation between the Cartesian coordinate system and the log-polar coordinate has been described above, the image in Cartesian coordinate can be flexibly sampled according to needs. The sampling image is divided into two parts, the central region with dense sampling, is oversampling area, the peripheral region with sparse sampling, is undersampling region. The size of the central dense region, also the over sampling area, is determined by the parameter $\mathrm{k}$. Set the radius of central dense region is $r$, as the boundary of central oversampling region and the peripheral undersampling region, and the sampling on this boundary is one-to-one match. The oversampling region is in the boundary, and the undersampling region is out of the boundary. The calculating formula of $\mathrm{r}$ is as follows:

$$
r=k \log k
$$

According to the needs, we can capture any interested position of image in Cartesian coordinate system, and compress the image purposefully. The interested region can be gazed with a high resolution, and the surrounding background can be recorded with a low resolution, as shown in Fig. 4 . Taking different image position as the center of log-polar coordinate, the original image is sampled, and then image restoration can be completed using sampling data. As is shown in Fig. 4, the region of interest has a high sampling resolution and its details are clear, the resolution of region of non interest is not high. So this sampling method is nonlinear and variable resolution.

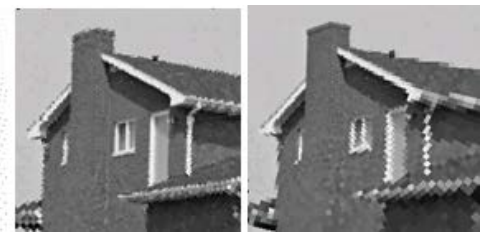

Fig. 4 Log-polar sampling image taking different image position as the center of log-polar coordinate

The experimental results of image compression and restored image

Record the lena image in log-polar coordinate, set the magnification factor $k=30$, the size of lena is $256 \times 256$ ( 65536 pixels), then the size of log-polar sampling image is $146 \times 188$. It can be calculated that the pixel record of single image decreases by 38088 pixels, and the image can be further compressed. Noting that the central region is oversampling area, it can be recorded directly by the image in Cartesian coordinate, while the peripheral undersampling area can be recorded in log-polar coordinate. As shown in Fig. 5, the lena can be expressed with two parts, one part is $188 \times 44$ pixels, another is $60 \times 60$ pixels. The total pixels of record are decreased by 53664 pixels, which accounted for only the $19 \%$ of the size of the original image. The compression ratio of image is $81 \%$, the most uninterested region of the image is greatly compressed. 


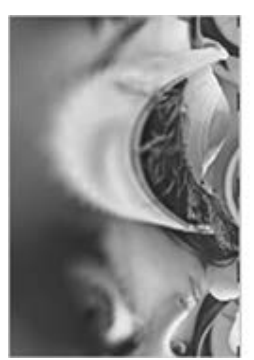

(a)

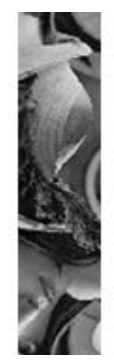

(b)

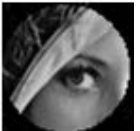

(c)

Fig. 5 (a)Image in log-polar coordinate (b)Undersampling area (c)Oversampling area

\section{Conclusions}

Inspiring by the human visual structure, two kinds of application of log-polar coordinate transform based on human visual structure model in image processing are introduced: image registration and image compression. The problems existing in traditional FMT are analyzed, and the new solutions are proposed in these two areas. In the field of image registration, Fourier-Mellin transformation based on log-polar coordinate is improved. In the field of image compression, a description method of image based on log-polar coordinate is proposed, which is a variable resolution storage and compression method, ensuring the region of interest not losing the details at the same time and reducing the resolution of background. The experimental results show that the correlation peak by improved method is higher, and the variable resolution image compression algorithm can compress image with a high compression ratio, which is suitable for image target tracking field.

\section{Acknowledgements}

This work is supported by Doctoral Found of QUST and the NKTRDP of Shandong province (2013YD01033).

\section{References}

[1] K. Schindler. Geometry and Construction of Straight Lines in Log-polar Images. Computer Vision and Image Understanding, pp.196 207(2006)

[2] A. Averbuch, R.R. Coifman, D.L. Donoho, M. Elad, M. Israeli. Fast and Accurate Polar Fourier Transform, Applied and Computational Harmonic Analysis, pp.145 167(2006)

[3] Y. Zhang, H.S. Qu, J. Zhao, Y.J. Wang. Log-polar matching for moving target attitude detection. Opto-electronic Engineering, Vol.34, No.6, pp.11-14(2007)

[4] R. Szeliski. Image Alignment and Stitching: A Tutorial[R]. Richard Szeliski Preliminary draft, Technical Report, MSR-TR-2004-92(2005)

[5] Z.X. Li, Y.B. Mao, Z.Q. Wang. A method of image mosaic using log-polar coordinate mapping[J]. Journal of Image and Graphics, Vol.10, No.1, pp.59-63(2005)

[6] H. Oh, A. Bilgin, and M.W. Marcellin. Visually lossless encoding for JPEG 2000, IEEE Transactions on Image Processing, Vol.22, No.1, pp.189-201(2013)

[7] D.R. Shen, J.L. Gong, X.P. Cao. Hyperspectral Imagery Compression Based on Linear Spectral Mixture Theory[J]. Transactions of Beijing Institute of Technology, Vol.30, No.1, pp.79-82(2010)

[8] Y.Y.Gao, J.F. Yang, X.L Ma, H.F. Chen. Interference image registration based on Fourier-Mellin algorithm. Optics and Precision Engineering, Vol.15, No.9, pp.1415-1420(2007)

[9] C.D. Kuglin, D.D. Hines. The phase correlation image alignment method]. IEEE Conference on Cybernetics and Society, pp.163-175(1975)

[10] F.J. Harris. On the Use of Windows for Harmonic Analysis with the Discrete Fourier Transform. Proceedings of the IEEE, Vol. 66, No.1(1978) 